\title{
Gerenciamento da cadeia de suprimentos de impressão e imagem com RFID
}

\author{
Edmilson Carneiro Moreira e José Wagner de O. Bezerra
}

\begin{abstract}
Resumo-Este trabalho foca na análise de viabilidade da identificação por rádio frequência, RFID, para ser utilizado no inventário de cartuchos de tinta e toners especificamente. Visando melhorar a gestão da cadeia de suprimentos para as empresas do setor de impressão e imagem, um armário utilizando RFID foi desenvolvido como prova de conceito para investigar a possibilidade de rastrear esses suprimentos usando o estado da arte do RFID. Os quadros e gráficos comparativos resumem os resultados de desempenho, levando este trabalho a conclusão sobre a capacidade de rastreamento de cartuchos de tinta e toners do armário inteligente.
\end{abstract}

Palavras-Chave-Sistemas de Telecomunicações, Redes sem Fio, RFID.

Abstract-This paper focus in feasibility analysis of radiofrequency identification, RFID, to be used in item level inventory of ink cartridges and toners specifically. In order to improve supply chain management and availability for companies in the printing and imaging business, a RFID enabled cabinet system was developed as a proof of concept to investigate the possibility of tracking those supplies using the state-of-the-art RFID. Comparative graphics summarize the performance results, leading this paper conclusion about ink cartridges and toners tracking capability of the cabinet system.

Keywords-Telecommunications Systems, Wireless Networks, RFID.

\section{INTRODUÇÃO}

Atualmente, o setor de impressão e imagem movimenta enormes quantias de dinheiro. A empresa que lidera este mercado teve um lucro de 28.456 milhões de dólares em 2006, sendo $59 \%$ em suprimentos. Uma grande parte do lucro em todos os segmentos da mesma empresa provém das vendas de cartuchos de tinta [1]. As vendas dos toners também estão aumentando devido ao crescimento do mercado de impressão a laser. Assim, uma gestão eficiente dessas cadeias de suprimentos é fundamental para aumentar a disponibilidade de cartuchos de tinta e toners, elevando as vendas desses produtos. RFID, uma tecnologia de identificação automática que usa ondas de rádio frequência [2], está surgindo como uma ferramenta poderosa para aumentar a eficiência da gestão da cadeia de abastecimento. Um excelente exemplo é a utilização de RFID para automatizar o processo de inventário de almoxarifados em empresas do setor de impressão e imagem. Este trabalho concentra seus esforços na concepção, implementação e avaliação de um protótipo de armário que utiliza RFID para rastrear automaticamente os cartuchos de tinta e toners nele armazenados, possibilitando uma gestão

Edmilson Carneiro Moreira e José Wagner de O. Bezerra, Instituto Atlântico, Fortaleza, Brasil, E-mails: edmilson@atlantico.com.br, wagner.bezerra@gmail.com mais eficiente da cadeia de abastecimento de ambos os itens. Primeiramente, definimos o sistema e seus requisitos. Em seguida, são selecionados os equipamentos de hardware a serem usados. Os testes preliminares visam nortear o projeto do armário e os de validaçao objetivam consolidar o que foi proposto. Por fim, é apresentada uma conclusão sobre o uso de RFID no rastreamento de toners e cartuchos de tinta, segundo os requisitos apresentados.

\section{O ARMÁRIO INTELIGENTE}

$\mathrm{O}$ armário inteligente permite um inventário extremamente preciso de cartuchos de tinta e toners, usando um processo automático, sendo a utilização da tecnologia RFID para esses tipos de produtos, os toners em especial, a sua principal inovação. Caso o inventário do armário mude, ações como reposição ou a verificação da data de validade, por exemplo, podem ser feitas automaticamente e imediatamente. Em resumo, o processo de leitura dos objetos a serem rastreados acontece quando um sistema computacional pede ao leitor para estabelecer a comunicação com as etiquetas eletrônicas na zona de leitura. $\mathrm{O}$ interrogador emite um sinal de RF através de suas antenas para as etiquetas que acompanham os itens, perguntando sobre seus números de identificação individuais. As etiquetas respondem os seus números exclusivos de volta para o leitor, tornando possível a identificação dos itens portadores de etiquetas.

\section{ESPECIFICAÇÕES INICIAIS}

A fim de estabelecer um ponto de partida para o desenvolvimento do armário, alguns requisitos foram estabelecidos. O primeiro deles é a utilização da norma ISO 180006C, também conhecido como EPC Gen2, que usa $915 \mathrm{MHz}$ como sua frequência central de funcionamento e a reflexão eletromagnética como seu mecanismo de acoplamento[2]. Essa escolha foi apoiada pela tendência da queda dos preços nas etiquetas da tecnologia Gen2 [3]. Outro requisito é a utilização de um modelo específico de armário com 1,5 m, 0,76 m e $0,54 \mathrm{~m}$ de altura, largura e profundidade, respectivamente. Pelo menos $50 \%$ das tentativas de leitura deveriam ter sucesso para considerar uma etiqueta presente na zona de leitura, sendo este critério baseado na figura TornOn Value estabelecida pela EPCglobal para determinar o alcance satisfatório de resposta de uma etiqueta ou grupo de etiquetas [4].

\section{ITENS RASTREADOS}

As características físicas dos itens a serem rastreados influenciam todas as variáveis no projeto de um sistema de RFID. 
Absorção, difração e reflexão são exemplos de perturbações no campo de rádio frequência [5]. Toners e cartuchos de tinta causam vários distúrbios em um campo de RF. Em primeiro lugar, há a reflexão do sinal de RF pelos toners, os quais apresentam uma grande quantidade de partes metálicas, e o pó do toner tem cerca de $40 \%$ de magnetita $\left(\mathrm{Fe}_{3} \mathrm{O}_{4}\right)$ [6] [7] [8] em sua composição, amplificando a capacidade de atenuar o sinal. Outro distúrbio é a atenuação do sinal de RF causada pela absorção de energia pela tinta líquida dos cartuchos de tinta que possuem um volume médio de $335 \mathrm{~cm}^{3}$. Esses fatores tornam o campo eletromagnético muito instável, apresentando sombras ou null fields de acordo com os tipos de toner e de como esses são colocados dentro do armário, tornando muito difícil que todas as etiquetas sejam alcançadas. A Figura 1 mostra a interação entre os itens com etiquetas e as ondas RF.

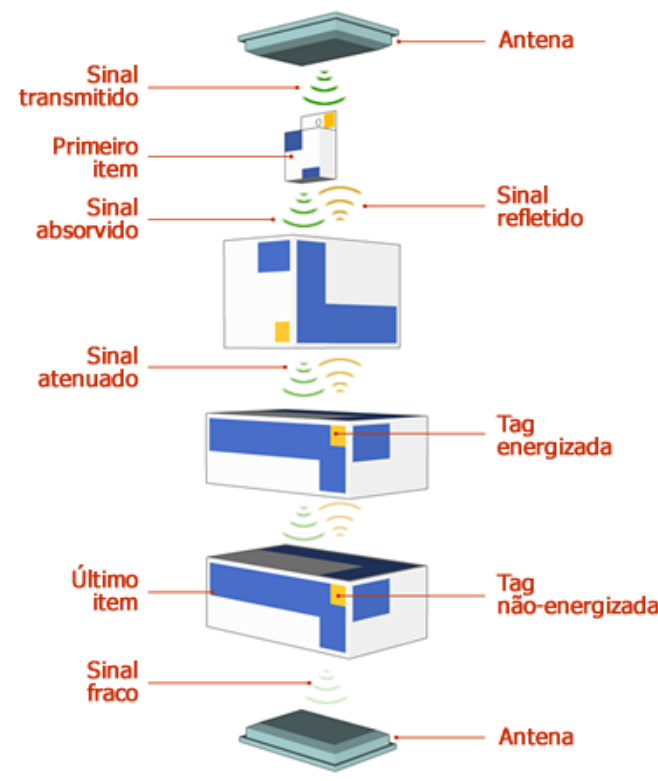

Fig. 1. Processo de atenuação do sinal de RF.

\section{Seleção dos equipamentos de RFID}

Vários pontos devem ser considerados para selecionar o conjunto mais adequado de equipamentos de RFID para uma aplicação específica, estando presentes em qualquer arquitetura três componentes fundamentais [2]. A parte inteligente do sistema é o interrogador ou leitor que controla o módulo de RF, bem como a comunicação com outros dispositivos, utilizando a porta serial e as interfaces de rede. Em sequência, estão as antenas, que transformam os sinais elétricos em ondas de rádio e vice-versa. O último é o transponder, etiqueta ou tag que realmente carrega a informação, sendo feita de uma antena e um CI eletrônico. Os critérios de seleção do hardware de RFID foram baseados em dois pontos básicos: a literatura disponível e a experiência da equipe de engenharia de rádio frequência do Instituto Atlântico. A EPC Certified Readers List [9] e um artigo de comparação de desempenho [10] foram as principais referências usadas nesta seleção do hardware de RFID. Para este estudo de caso, foram testadas 3 interrogadores com as suas próprias antenas e 2 tipos de etiquetas, perfazendo um total de 6 conjuntos de hardware. Vale ressaltar que todo o hardware utilizado neste trabalho é composto de equipamentos existentes no mercado.

\section{Metodologia dos testes}

O escopo dos testes foi a validação da viabilidade do monitoramento de toners e cartuchos de tinta, usando o padrão ISO 18000-6C. Testes preliminares foram realizados para reunir dados iniciais a fim de definir um cenário que indicasse a possibilidade de efetivamente rastrear esses itens, usando RFID. Os procedimentos de testes aplicados neste trabalho foram adaptados a partir dos utilizados em [11]. Todos os testes foram realizados no mesmo ambiente. A temperatura da sala foi mantida entre 22 e 28 graus Celsius e umidade relativa do ar foi de cerca de $80 \%$. Havia vários outros dispositivos elétricos e eletrônicos funcionando no laboratório, tais como telefones, computadores, telefones celulares e aparelhos de arcondicionado.

\section{TESTES PRELIMINARES E O PROJETO DO ARMÁRIO INTELIGENTE}

Alguns testes preliminares foram feitos com a intenção de selecionar a polarização das antenas, o posicionamento das etiquetas nos toners e nos cartuchos de tinta, bem como a disposição desses itens no armário. Uma vez decidido o posicionamento nos itens e a polarização das antenas do leitor, a arrumação dos itens no armário foi investigada. A configuração mais intuitiva é a absolutamente aleatória, onde os produtos são colocados dentro do móvel sem nenhuma restrição. Os testes com esse arranjo mostraram que era praticamente impossível detectar todas as etiquetas no armário devido principalmente às características físicas dos itens monitorados. Já que esses testes demonstraram que o sistema foi incapaz de detectar todas as etiquetas associadas à toners e cartuchos de tinta, na arrumação completamente aleatória, é necessária uma nova abordagem. Ensaios com arranjos controlados foram propostos com a intensão de alcançar números de inventário mais precisos. Esta abordagem implica num posicionamento específico das etiquetas e, consequentemente, um reposicionamento das antenas do leitor de RFID. Com o objetivo de superar as dificuldades impostas pelas características físicas dos itens, um posicionamento controlado de toners e cartuchos de tinta foi feito com o estabelecimento de duas regras. Primeiramente, todas as etiquetas dos toners devem estar em um único plano geométrico no interior do armário. Esse arranjo permitiu que todas as etiquetas ficassem visíveis para as antenas. A segunda regra dita que todos os cartuchos de tinta devem ser suspensos em ganchos de acrílico, garantindo que a orientação das etiquetas destes itens sejam mantidas. Os testes preliminares com arranjos controlados apresentaram desempenho significativamente melhor do que o aleatório. Então, esse foi selecionado para passar pelos testes de validação. Seguindo as regras e requisitos citados anteriormente, a proposta do projeto do armário é apresentada na Figura 2. 


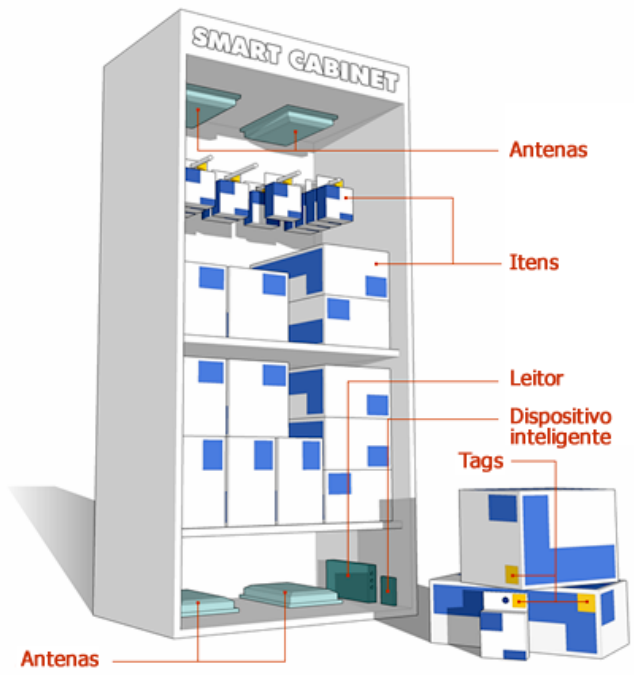

Fig. 2. Layout do armário.

\section{Testes de VALIDAÇÃo}

Os testes preliminares definiram o projeto do armário e produziram uma gama de informações que orientaram este trabalho a definir o melhor cenário - a disposição controlada desses itens dentro do móvel - para viabilizar o monitoramento de cartuchos de tinta e toners. Definido o escopo destes testes, a forma como estes devem ser realizados entrou em questão. Devido a um número quase infinito de possíveis arranjos no armário, um número finito dessas configurações deve ser estabelecido. Empiricamente, dez arranjos pareciam uma escolha razoável já que este trabalho foca um estudo de viabilidade. Assim, dez possíveis arranjos devem ser feitos e documentados, com o objetivo de repeti-los por várias baterias de testes. Esses foram obtidos através da colocação de uma quantidade máxima de cartuchos de tinta nos ganchos do armário e da inserção dos toners selecionados aleatoriamente, respeitando as regras anteriormente citadas, até que o armário esteja populado e o arranjo documentado. $\mathrm{O}$ procedimento de teste começou com a colocação das quatro antenas do leitor no armário. Em sequência, o interrogador foi ligado e conectado a um computador executando um software de teste. Um desses arranjos documentados é montado. O software de teste é executado cem vezes e o número de leituras feitas é registrado para cada etiqueta, consequentemente, o item que ela está anexada. $\mathrm{O}$ teste foi aplicado para cada um dos arranjos documentados e para cada combinação possível de leitores, suas antenas e tipos de etiquetas. Os seis conjuntos de hardware foram testados com todos os arranjos documentados. O objetivo deste teste foi investigar o nível de precisão na leitura de um conjunto de arranjos controlados de itens, de acordo com o número de etiquetas eletrônicas detectadas na zona de leitura. Os resultados destes testes mostraram que forçar o posicionamento das etiquetas melhora bastante a precisão dos inventários. A Figura 3 mostra o percentual de detecção de etiquetas neste cenário.

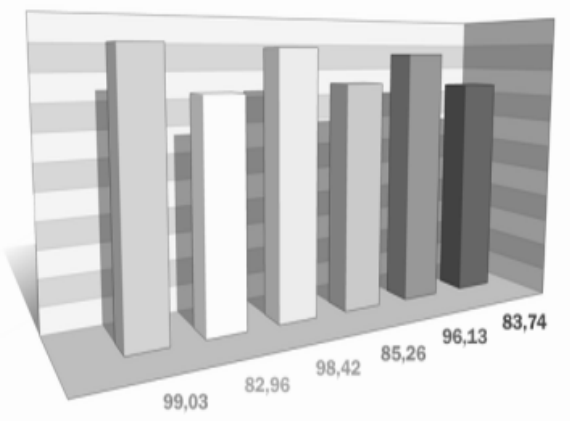

Conjunto de hardware 01

Conjunto de hardware 02

Conjunto de hardware 03

Conjunto de hardware 04

Conjunto de hardware 05

Conjunto de hardware 06

Fig. 3. Taxas de detecção de etiquetas

\section{OS RESULTADOS DOS TESTES E MELHORAMENTOS}

Os resultados apresentados na Figura 3 indicam que o modelo proposto não pode atingir $100 \%$ de detecção de etiquetas, ainda. Destarte, foi necessária uma análise mais profunda, objetivando o aperfeiçoamento do sistema para que a detecção de todas as etiquetas seja uma realidade. Depois de várias tentativas, utilizando o conjunto de hardware que obteve os melhores resultados com um sutil ajuste neste sistema de RFID, a detecção de todas as etiquetas nos 10 arranjos documentados foi alcançada, indicando viabilidade até então. Essa adequação consiste, principalmente, no reajuste do posicionamento do plano de etiquetas dos toners que deverá receber uma potência irradiada maior que a anterior a este reposicionamento. Os resultados da última tentativa estão na Figura 4.

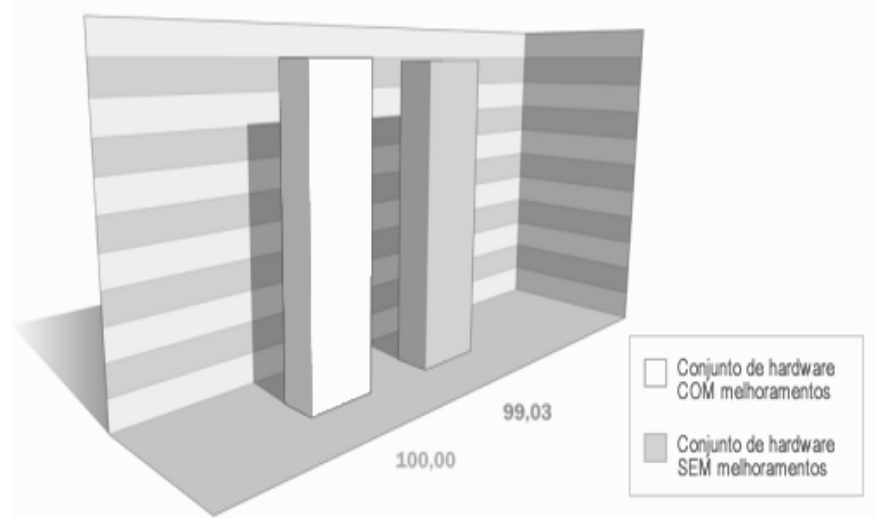

Fig. 4. Taxas de detecção de etiquetas.

\section{Conclusões}

O protótipo de armário inteligente apresenta um ambiente extremamente complexo para utilizar identificação por rádio frequência, usando o padrão ISO 18000-6C. Existem dois grandes problemas. O primeiro é altamente relacionado com as características físicas dos itens. A grande quantidade de metais presentes em um toner reflete o sinal de RF, criando sombras ou null fields em toda a zona de leitura do móvel. Os cartuchos de tinta perturbam as ondas de rádio frequência com menor intensidade, pois a quantidade de componentes atenuantes é consideravelmente menor do que nos toners. $\mathrm{O}$ segundo se refere 
à impossibilidade de determinar a localização e a orientação das etiquetas dos produtos no interior do armário. As regras de posicionamento diminuíram drasticamente o número de etiquetas não detectadas, mas nos testes de validação sem um ajuste fino do sistema de RFID, nenhum conjunto de hardware foi capaz de atingir $100 \%$ de detecção das etiqueta em todos os arranjos documentados. Após os testes de validação, a detecção de todas as etiquetas veio como resultado de uma profunda compreensão do problema, tornando o ajuste do hardware de RFID possível. Ainda que os resultados finais indiquem a viabilidade do uso de RFID para rastrear toners e cartuchos de tinta, são sugeridos futuros ciclos de testes com vários arranjos para garantir a estabilidade do sistema. Além disso, todos os conjuntos de hardware de RFID usados nos testes de validação devem ser testados com os mesmos ajustes finos feitos na bem sucedida tentativa deste trabalho. Portanto, a utilização de RFID para monitorar os cartuchos e toners é viável com o uso de algumas regras de posicionamento e um sistema de RFID muito bem ajustado para esta problemática específica.

\section{REFERÊNCIAS}

[1] Wikinvest.com, Hewlett Packard Company, http://www.wikinvest.com/stock/Hewlett-Packard_Company_(HPQ), 2008.

[2] Klaus Finkenzeller, RFID Handbook: Fundamentals and Applications in Contactless Smart Cards and Identification, John Wiley and Sons, Inc., Second Edition, 2003.

[3] ADT/Tyco Fire \& Security, Alien, Impinj, Intel, Symbol and Xterprise, RFID and UHF: A Prescription for RFID Success in the Pharmaceutical Industry, http://www.rfidjournal.com/whitepapers/download/127, Julho 2006.

[4] Dennis E. Brown, RFID Implementation, McGraw-Hill, First edition, 2007

[5] Paul Sanghera, RFID+ Study Guide and Practice Exam, Syngress Publishing, Inc., First Edition, 2007.

[6] Lexmark Inc., Material Safety Data Sheet http://www.lexmark.com/pdf/msds/en/laser_mono/11A4097.pdf, 2003.

[7] Hewlett Packard Company., Material Safety Data Sheet, http://www.hp.com/hpinfo/globalcitizenship/environment/productdata/ pdf/lj_c8543x-Xc_us_eng_v10.pdf, 2003.

[8] Xerox Corporation., Material Safety Data Sheet, http://web.grcc.cc.mi.us/Pr/msds/financialaid/XeroxCopyTonerXerox8000series.pdf, 2002.

[9] EPCglobal Inc., EPCglobal Certified UHF GEN2 Reader/Readers Modules, http://www.epcglobalinc.org/certification/hw_cert/, 2009.

[10] Daniel Deavours, Choosing an EPC GEN2 Interrogator, RFID Journal, Junho 2006

[11] Edmilson Carneiro Moreira, José Wagner de O. Bezerra e Marcelo Ferreira Sousa, A RFID Device to increase shelf replenishment efficiency, II RFID Community Workshop, 2007. 